\title{
Compatibilizando estudios y familia: Ser estudiante y a la vez madre o padre [Combining studies with family:
}

Being a student and a parent at the same time] Diego Álvarez Moral ${ }^{(1)}$, Catalina Guerra Maldonado(D), Karen Tapia Sepúlveda (1) \& Gonzalo Varas González(D)

Universidad de Chile

\begin{abstract}
Resumen
En este artículo se abordan las estrategias de compatibilización de actividades académicas y familiares en estudiantes padres y madres universitarias, y sus repercusiones a nivel subjetivo, en términos de salud mental y percepción en el desempeño de sus roles. Para ello, se realizaron entrevistas semiestructuradas a estudiantes madres y padres de la Universidad de Chile pertenecientes a los cinco campus de la institución, las cuales se analizaron mediante análisis de discurso. Los principales resultados indican presencia de jornadas largas, extenuantes y variadas, las que son sobrellevadas por medio de estrategias caracterizadas por una notoria autoexigencia y despliegue de redes de apoyo. Estas tienen consecuencias negativas en la salud mental y generan percepciones no tradicionales sobre la juventud, la maternidad y la paternidad. El estudio entrega insumos para el fomento de espacios y reglamentos universitarios más amigables para la población estudiantil materno-paterna.
\end{abstract}

Palabras clave: cuidados, maternidad, paternidad, estrategias de compatibilización.

\begin{abstract}
This article addresses the strategies adopted by university students who are also parents as they combine academic and family activities, exploring the repercussions of these strategies at a subjective level in terms of mental health and perceptions of their performance in the two roles. Semi-structured interviews were applied to students who are parents at the five University of Chile campuses. Results were interpreted using discourse analysis and reveal long, exhausting, and varied daily routines involving considerable personal demand and reliance on support networks. These strategies were found to have negative consequences in terms of mental health and to generate non-traditional perceptions of youth and parenthood. The study proposes foundations for the promotion of university spaces and guidelines that are more sympathetic to students who are also parents.
\end{abstract}

Keywords: care, motherhood, fatherhood, parenthood, combination strategies.

Contacto: La comunicación sobre este artículo debe ser enviada a Diego Álvarez Moral, email diego.alvarez.m@ug.uchile.cl

Financiamiento: Esta investigación contó con apoyo de la Dirección de Igualdad de Género (DIGEN) de la Universidad de Chile y la Federación de Estudiantes de la Universidad de Chile (FECH). 


\section{INTRODUCCIÓN}

Los modos en que padres y madres lidian con las múltiples responsabilidades a las que se ven expuestos suelen observarse como desafíos personales. Habitualmente, las instituciones en las que se insertan tienden a escindir su dimensión personal y familiar de la pública. Un ejemplo de ello son las universidades chilenas, en tanto la relación que establecen con sus estudiantes suele limitarse a lo académico. Si bien los apoyos económicos que brindan los diversos establecimientos de educación superior a sus alumnos/as protegen algunas condiciones de subsistencia que permiten la retención del estudiantado (Canales \& De los Ríos, 2009). No obstante, en su funcionamiento cotidiano, impera una distinción entre lo público (vida universitaria) y lo privado (cuidados y crianza).

Esta escisión se encuentra en la base de los procesos de modernización universitaria. En términos históricos, la universidad transita de un espíritu medieval a uno moderno lo que, en términos de ideario y funcionamiento, significa el tránsito de una moral jurídico-eclesiástica a una de carácter político-económica (Clark, 2006). Esto se condice con la clásica distinción público-privado de la racionalidad moderna, habitualmente tratado en el ámbito de la política y el trabajo (Federici, 2013; Pateman, 1995), y también se configura en el espacio académico, abstrayendo los procesos de formación educacional del resto de las esferas vitales de las personas (Clark, 2006).

Dicha concepción se tensiona en el caso de estudiantes padres/madres donde la conciliación entre familia y estudios es dificultosa de abordar, en tanto las instituciones apuntan a un alumnado que dedique la mayor parte de su tiempo a los estudios. Las universidades sólo recientemente han comenzado a cuestionar su propia participación en el apoyo a sus estudiantes en la medida que han experimentado procesos de ampliación y diversificación de la matrícula, coincidiendo con un perfil que dispone de cierto nivel importante de capital cultural, social y económico (Araneda, Gairín, Pedraja-Rejas \& Rodríguez-Ponce, 2018; Donoso \& Schiefelbein, 2007).

La distinción entre lo público y lo privado configura también la división sexual del trabajo. Persiste una marcada feminización de las actividades privadas, especialmente aquellas ligadas a lo doméstico y los cuidados. Por lo demás, crecientemente la mujer participa de los espacios públicos a través de su mayor incorporación a espacios como el trabajo o la universidad, pero no se ha desarrollado un movimiento equivalente de parte de los hombres hacia las responsabilidades del ámbito privado. La escisión público-privado, se presenta aquí como una distinción entre los tiempos del cuidado y los tiempos del estudio (sin considerar otras responsabilidades). El tiempo es, precisamente, la unidad fundamental a través de la cual el conflicto estudio-familia se cristaliza. Esto ha sido bien tratado por los estudios en usos del tiempo y los debates del conflicto trabajofamilia.

La sobre exigencia de la población femenina producto del trabajo que aporta tanto en las 
esferas públicas como privadas se encuentra a la base de una marcada crisis de los cuidados, aumentando su complejidad y requiriendo articular complejas estrategias que permitan maniobrar el cumplimiento de todos los roles sociales (Arriagada, 2010). Estas transformaciones tienen un importante impacto en cómo madres y padres viven su proceso de (re)construcción de identidad (Rivas, 2010), por lo que tener en cuenta dichos cambios es fundamental. Lo anterior también exige a las organizaciones y al Estado repensar las opciones que ponen a disposición para lograr un equilibrio entre la vida familiar y laboral. Las universidades han presentado escasos avances hacia una perspectiva similar, considerando el aumento de la matrícula femenina y mayor apertura a un estudiantado crecientemente diverso (SIES, 2019).

El cuidado, como la "gestión y generación de recursos para el mantenimiento cotidiano de la vida y la salud; la provisión diaria de bienestar físico y emocional, que satisfacen las necesidades de las personas a lo largo de todo el ciclo vital" (Arriagada, 2010, p. 58) apela a una presencia completa de la persona cuidadora. Esto puede darse de manera directa (estar presente físicamente en las actividades de cuidado) o indirecta (no presente) (Arriagada, 2010). En ambos casos, sin embargo, los cuidados se caracterizan por no poseer un horario delimitado y requerir atención constante de quienes lo proveen, además de una disposición mental y emocional permanente (Carrasco, 2013), relevando tanto su materialidad como su subjetividad. Lo anterior supone una gestión de servicios y recursos que permitan tanto a quien desempeña el rol de cuidador como al beneficiario/a, estar sanos/as y vivir en un ambiente propicio. En el caso de estudiantes madres y padres, ello exige desplegar distintas estrategias con el fin de compatibilizar sus distintas responsabilidades, las cuales implican la gestión parcialmente controlada de su tiempo y redes de apoyo.

Los cuidados no sólo son necesarios para la reproducción física de la vida, sino que están directamente relacionados con el bienestar humano, tanto de quienes cuidan como de quienes son cuidados (Carrasco, 2013). No obstante, "las concepciones culturales consideran que la reproducción social es una responsabilidad femenina y no una necesidad" (Universidad de Chile, 2018, p.9), siendo tareas que se llevan a cabo mediante la donación de tiempo y energía por parte de las mujeres en su mayoría, con escaso reconocimiento social y nula remuneración. Por lo tanto, plantea un escenario de alta exigencia organizacional de la vida cotidiana, pero sin una gratificación o retribución equivalente, constituyendo una posible fuente de malestares a nivel de salud mental y autopercepción.

Las dificultades surgidas del conflicto entre los estudios y el cuidado exigen a estudiantes padres y madres el despliegue de estrategias que permitan un manejo óptimo del tiempo (PNUD, 2012). Ante una situación de escaso tiempo libre, las estrategias de gestión del tiempo y de compatibilización de roles familiares y estudiantiles aparecen como una exigencia para la adaptabilidad. Entendemos, entonces, estas estrategias como el despliegue de la subjetividad y las redes disponibles en contextos objetivamente complejos (Wilkis, 2004) y en los cuales estas personas se ven obligadas a cumplir distintas obligaciones-a 
veces contrapuestas entre sí-por los roles que tienen asignados en base a su condición, operando para optimizar el uso del tiempo (PNUD, 2012). Bajo un contexto de crisis generalizada de los cuidados, Arriagada (2010) identifica cinco estrategias para conciliar trabajo y familia, realizando un símil con los estudios en este caso. (a) La reducción de los objetivos, que consiste en una menor aspiración en el cumplimiento de objetivos; (b) la delegación, que consiste en buscar a otra persona que se haga cargo de tareas asociadas a alguno de los roles; (c) la secuencialización que consiste en alternar los tiempos de estudio con la vida familiar; (d) la derivación, que se refiere a buscar soluciones para las tareas de cuidados en el mercado (cuidados remunerados) y; (e) el reparto de tareas, que es generar una organización con el padre o la madre del hijo/a que permita que quien estudia no cargue con todas las labores de cuidado.

Actualmente, Chile posee una marcada tendencia a la desinstitucionalización de la familia que ha generado mayor diversidad en los tipos de familias, monoparentales o con jefatura femenina del hogar, además del reconocimiento legal de los hijos nacidos fuera del matrimonio (Valdés, 2004). Según el Instituto Nacional de Estadística de Chile (INE, 2017), el embarazo adolescente se redujo de 89.1 por cada 100,000 en 1963 a 40.6 por cada 100.000 en 2015. Pese a esto, un 55\% de los jóvenes chilenos ha experimentado un embarazo no planificado antes de los 20 años y un $32 \%$ de la población entre 15 y 19 años tiene al menos un hijo (Dides \& Fernández, 2016).

La Ley General de Educación plantea al proceso de aprendizaje escolar como un derecho que debe estar garantizado, declarándose así la ley 20.370 en 2010: "El embarazo y la maternidad no debe presentarse como limitaciones para ingresar o continuar en el proceso educativo". Estas situaciones han ido siendo visibilizadas por las universidades chilenas. La Universidad de Chile fue una de las primeras universidades en movilizarse a favor de las demandas levantadas a raíz las movilizaciones estudiantiles feministas de 2018 que, entre otras cosas, buscaban la reivindicación de los derechos de las y los estudiantes con hijos (Huerta, 2018). A nivel de la experiencia de estos estudiantes, Castañeda (2015) ha dado cuenta de la necesidad que tienen de desplegar estrategias para conjugar estudios y responsabilidades familiares. Entre estas estrategias, destaca el rol de las redes de apoyo familiares, cuyo soporte determina la continuidad de estudios y la mantención económica. Para las mujeres la noticia de la maternidad es disruptiva y crítica, en tanto se encuentran fuertemente determinadas por roles de género patriarcales y, con ello, quienes más deben realizar sacrificios en pos del cuidado y en desmedro de sus proyectos personales. Esto último lleva a que las estudiantes que son madres y las parejas de los estudiantes varones retrasen sus estudios en mayor medida que los estudiantes padres, los cuales en general compatibilizan trabajo y estudio, pero en los casos en que asumen responsabilidades de cuidado y crianza, comienzan a presentar los mismos problemas de compatibilización.

Esta investigación retoma parte de las preocupaciones de Castañeda (2015), pero bajo un contexto de creciente preocupación institucional por parte de la Universidad de Chile como respuesta a las movilizaciones feministas. Esto la vuelve un foco 
interesante de análisis para la situación de las maternidades y paternidades universitarias, pues plantea perspectivas a tomar en cuenta para otras instituciones que busquen aplicar reglamentos en torno a cuidados en la paternidad/maternidad. Adicionalmente, la Universidad de Chile constituye uno de los principales casos de universidad tradicional de alta exigencia en Chile, lo que permite tensionar el perfil tradicional del estudiante universitario a través del carácter disruptivo de la maternidad y paternidad. Así, el objetivo de esta investigación es analizar las estrategias de compatibilización de responsabilidades en la vida personal de estudiantes padres y madres, en el contexto de la implementación de la política de corresponsabilidad social en los cuidados de la Universidad de Chile.

\section{MÉTODO}

El estudio se sitúa desde una perspectiva cualitativa (Strauss \& Corbin, 2002) pues permite la indagación holística y en mayor profundidad del objeto de investigación al buscar aprehender la percepción de los actores en relación al contexto en el que se desenvuelven y las redes con que interactúan en su proceso de compatibilización de responsabilidades. Para la producción de datos se usaron entrevistas semi estructuradas a estudiantes padres y madres.

La población de estudio fueron los estudiantes padres y madres con matrícula vigente entre los años 2013 y 2019 en las diversas facultades e institutos de la Universidad de Chile en calidad de estudiantes regulares que, conforme a los datos estadísticos oficiales (Dirección de Bienestar y Desarrollo Estudiantil, 2019) corresponden a 335 jóvenes (224 mujeres y 111 hombres).

La muestra fue construida atendiendo a un carácter estructural y estuvo compuesta por madres y padres de cada campus (i.e., Eloísa Díaz, Andrés Bello, Beauchef, Juan Gómez Millas y Antumapu). De este modo, se alcanzó un total de 16 entrevistas.
La composición de la muestra final se describe en la Tabla 1. A esta se suma la realización de un grupo focal con madres de todos los campus. La forma de contacto con las personas que conformaron la muestra fueron cuatro. Primero, se revisaron las bases de datos de estudiantes con hijos que postularon a la beca de apoyo preescolar (BAE), la base de datos de quienes respondieron la Encuesta de Padres y Madres Universitarios y el censo de estudiantes madres y padres realizado el 2019. Se contactó además a organizaciones sociales y dirigentes estudiantiles que pudieran entregar el contacto de casos por conocimiento común, para efectuar a su vez la estrategia de bola de nieve a los mismos casos estudiados, consultando por otros alumnos padres. El acceso a todas las bases de datos se facilitó con el compromiso de confidencialidad y anonimato de los casos, y estos fueron utilizados únicamente con fines investigativos. La participación de los estudiantes en las entrevistas fue mediada por la firma de un consentimiento informado, asegurando su confidencialidad. 
Tabla 1. Composición de la muestra según Campus (Facultades) y sexo.

\begin{tabular}{lccc}
\hline Campus (Facultades) & & Madres & Padres \\
\hline Eloísa Díaz (Facultad de Salud) & & 1 & 1 \\
Andrés Bello (Facultades de: Arquitectura y Urbanismo; Derecho; & & 5 & 1 \\
$\begin{array}{l}\text { Economía y Negocios) } \\
\text { Beauchef (Facultad de Ciencias Físicas y Matemáticas) }\end{array}$ & & 1 \\
$\begin{array}{l}\text { Juan Gómez Millas (Facultades de: Ciencias Sociales; Comunicación } \\
\text { e Imagen; Ciencias; y Filosofía y Humanidades) }\end{array}$ & 3 & 2 \\
Antumapu (Facultad de Agronomía) & & 1 & 0 \\
\hline Total & & 11 & 5 \\
\hline
\end{tabular}

La técnica de producción de información escogida fue la entrevista en profundidad semiestructurada pues permite conocer en profundidad las experiencias personales de los y las entrevistadas, permitiéndoles expresar libremente "sus valoraciones, motivaciones, deseos, creencias y esquemas de interpretación que los propios sujetos bajo estudio portan y actualizan durante la interacción de entrevista" (Gaínza, 2006, p. 220). Se eligió el formato "semi estructurado" de entrevistas, debido a su flexibilidad, basada en una guía de asuntos o preguntas donde el entrevistador tiene la libertad de introducir preguntas adicionales para precisar conceptos u obtener mayor información sobre los temas deseados (Hernández, Fernández \& Baptista, 2010). Este formato permitió generar una pauta de preguntas que guiaron el curso de la entrevista, con el fin de alcanzar los objetivos de la investigación. Los tópicos de conversación de las entrevistas se basaron tres dimensiones: (a) Reglamento de corresponsabilidad de la universidad (conocimiento del reglamento, uso y críticas a éste); (b) redes de apoyo a la paternidad-maternidad externas a la universidad (reparto de tareas, derivación/delegación) y; (c) estrategias de compatibilización de responsabilidades paterno-maternas en la vida personal (secuencialización, uso del tiempo, implicancias subjetivas, percepción propia y del entorno, y reducción de objetivos).

Las entrevistas fueron analizadas mediante análisis de discurso que es un método y, a la vez, una teoría sobre los hechos simbólicos comprendidos en el discurso de los entrevistados. Esta estrategia de análisis es un procedimiento de análisis de textos y de representaciones, que da cuenta de la estructura que organiza los sentidos del texto (Martinic, 2006). Esto permite establecer relaciones, fracturas e intersecciones entre las variables, entregando la posibilidad de establecer cómo se configuran las relaciones intersubjetivas en los y las estudiantes respecto a los roles que desempeñan, y permitiendo reconstruir dichas significancias en tres dimensiones: su contenido informacional, su estructura y su dimensión normativa (distribución de valorizaciones) (Moscovici, 1961). De esta manera se conecta el texto en su carácter semántico con las condiciones sociales que lo producen y transmiten (Martinic, 2006), siendo a nuestro juicio el método más provechoso para el análisis de información. 


\section{RESULTADOS}

Gestión del tiempo

Los ritmos del día a día de los estudiantes entrevistados se ordenan en función de las exigencias del cuidado, las responsabilidades académicas, domésticas y laborales. Se observan tres regularidades. En primer lugar, se presentan jornadas de actividad muy largas y variadas en contenido. Los días parten muy temprano para llevar al niño o niña al jardín infantil, sala cuna, o colegio, liberándose un tiempo para actividades académicas, como asistir a clases o estudiar. Los tiempos de socialización se dan principalmente en el almuerzo con compañeros de facultad, y el resto del día se destina a labores de cuidado hasta que sus hijos se duermen. Sin embargo, con frecuencia este tiempo liberado se destina al estudio:

Me levantaba muy temprano, levantaba a mi hija después, la iba a dejar al jardín. Del jardín me iba a la U [...] y todo el día porque tenía jornada completa y cuando saliera, ir al tiro a la casa, e ir a buscarla al jardín. Y de ahí no hacer nada de la U hasta que se quedara dormida [...] hacía todo en la noche" (Alumna, Campus Juan Gómez Millas - Facultad de Comunicación).

Este tipo de organización horaria genera gran cansancio y la sensación de una imposibilidad de darle a las actividades académicas el tiempo suficiente, sin considerar a los estudiantes que además cuentan con un trabajo remunerado. Una segunda regularidad es que las actividades diarias están organizadas en función de los tiempos del cuidado: "Entonces el [nombre del hijo] yo creo que ordena como un poco la vida. Las guaguas ordenan la vida de los papás" (Alumno, Campus Andrés Bello - Facultad de Derecho). Para las estudiantes los tiempos de los cuidados son difíciles de transar, aunque existan redes de apoyo: "Lo que más tiempo me quita, si se puede decir así, es el tiempo que dedico a estar con mi hijo." (Alumna, Campus Andrés Bello - Facultad de Derecho). El tercer punto refiere a la escasez de tiempo libre, pues prácticamente todo el tiempo es utilizado en responsabilidades universitarias o parentales. Esto deja escasos momentos de tiempo libre y/o para satisfacer sus necesidades básicas: "Corriendo mucho y durmiendo poco. Desde que entré a la U son las dos premisas de mi vida. Correr mucho y dormir poco" (Alumna, Campus Andrés Bello - Facultad de Arquitectura y Urbanismo).

Las realidades laborales de los estudiantes entrevistados son variadas, desde trabajos formales a informales. Este último tipo de trabajo les permite tener más control sobre sus tiempos, pero a la vez genera problemas a la hora de justificarse en caso de acogerse a las flexibilidades académicas que ofrece la universidad. Por otra parte, los trabajos formales tienden a realizarse durante los fines de semana, lo que genera la sensación de no poder descansar y tener poco tiempo para la crianza y las labores académicas. Una importante cantidad de estudiantes cuenta con apoyo económico familiar: "Mi familia me dio esa holgura de que iban a hacer un esfuerzo y yo podría no trabajar" (Alumna, Campus Andrés Bello-Facultad de Derecho), debido a la sobrecarga de responsabilidades y las 
consecuencias negativas en el rendimiento académico que esto trae consigo: "En el momento en que yo trabajaba, mi rendimiento académico se fue como, muy abajo" (Alumno, Campus Juan Gómez Millas Facultad de Ciencias).

La mayor parte sugiere que las responsabilidades del cuidado, ya sea directo o indirecto, involucran un importante desgaste físico o mental. Estudiantes de regiones son un ejemplo de cuidadores indirectos, pues pasan menos tiempo físicamente junto sus hijos, pero mantienen preocupación constante en torno a sus responsabilidades de cuidado y crianza: "Como yo soy de Rancagua normalmente toda la semana la pasó aquí y el fin de semana es para mi hijo. Entonces, tengo que ordenar mi tiempo de terminar todo lo que sea la universidad durante la semana, y el fin de semana de dedicarlo solamente a mi hijo" (Alumna, Campus Beauchef - Facultad de Ciencias Físicas y Matemáticas).

Ante cada una de estas situaciones, estudiantes padres y madres despliegan distintas estrategias con el fin de abordar todas sus responsabilidades. Por ejemplo, quienes mayormente expresan la necesidad de realizar cambios drásticos en el uso de su tiempo son las madres, mostrando mayor aceptación a retrasarse en sus carreras, mientras que padres tienden a manifestar la necesidad de terminarlas rápido para la manutención de sus hijos. Las estrategias de los estudiantes padres tienden a orientarse hacia la obtención de títulos profesionales mientras ejercen sus labores de crianza, a lo que se suma, en ocasiones, actividades laborales durante la semana, mientras que las estrategias de las estudiantes madres se orientan hacia priorizar sus labores de crianza por sobre su desempeño universitario, retrasando su ingreso al mundo laboral. Sin embargo, en ambos casos se observa como sus responsabilidades priman por sobre el ejercicio de tiempo libre o la realización de actividades relacionadas con necesidades básicas, tales como alimentación y descanso.

Gran parte de las personas señala repercusiones negativas en su salud mental ante la dificultad de compatibilizar responsabilidades a partir de la dificultad de adaptar los tiempos, redes y recursos. Además, hay una dimensión emocional referida a la necesidad de compartir con sus hijos/as y viceversa, la cual se cubre parcialmente, por falta de tiempo, o de forma ambigua al estar haciendo varias cosas a la vez, lo que estaría relacionado directamente con la calidad de vida y bienestar humano de quienes cuidan:

Me sentía súper culpable, y lloré mucho e incluso tuve depresión en ese tiempo por sentirme culpable de que mi hijo no tenía la culpa de que yo lo tuviera que traer a la universidad a esa hora de la mañana a pasar frío (Alumna, Campus Juan Gómez Millas - Facultad de Ciencias Sociales). Asimismo, se encontraron relatos sobre problemáticas psicológicas y de salud mental respecto a la percepción propia y de su entorno sobre sus roles de género en la crianza. Las estudiantes perciben que la paternidad responsable se reconoce y celebra, mientras que la maternidad se entiende como un deber y el entorno se atribuye mayores potestades para opinar, exigir, y responsabilizar:

Como que a los hombres no le piden mucho, así como que ¡Ay! si cambia un pañal es bacán, así es jel mejor papá del mundo!, pero a una como que le cuestionan todo [...] como que siempre lo puedo hacer mejor y, y cuesta caleta (Alumna, Campus Juan Gómez Millas - Facultad de Filosofía y Humanidades). 
Esta situación tiende a provocar sentimientos de ansiedad en las mujeres entrevistadas. Así mismo, se puede identificar una relación contradictoria de la tríada mujer-feminismo-maternidad en citas como la siguiente:

Yo creo que prima [el rol de madre], pero quisiera que no fuera así. Como que al final mi rol de madre es el principal de mi persona, pero al menos yo no quiero y no estoy dispuesta a que termine siendo lo único que soy [...] es lo que soy constantemente y casi todo el tiempo, pero no es lo que me define ni me representa, porque si fuera por eso será como, no sé, una mujer, pero no una mamá" (Alumna, Campus Juan Gómez Millas - Facultad de Filosofía y Humanidades).

Mientras se reconoce que la maternidad tiende a ser el rol preponderante, las estudiantes buscan compatibilizar dentro de lo posible con otras actividades, y se plantean reflexiones en torno a la importancia de ser mujeres realizadas para dar un buen ejemplo, poniendo estas reflexiones en discusión con roles de género tradicionales. También persisten concepciones patriarcales respecto a la paternidad puestas en tensión. Priorizar una "paternidad presente" por sobre una proveedora en términos económicos, validarse como padres cuando se pertenece al colectivo LGBTQ+ o cuando se es más joven en edad que la pareja, son algunas de las reivindicaciones observadas de parte de estudiantes padres de la muestra:

No me creen cachai, como que igual existe como todo este ámbito porque yo soy... gay [...] así que del momento en que me conocen, jamás piensan que soy papá, y tengo que pasar por todo este proceso de ¿Cómo pasó? ¿Y te gustaban las mujeres antes? ¿Y ahora no te gustan las mujeres? (Alumno, Campus Juan Gómez Millas).
Las personas entrevistadas señalaron diversos malestares, tales como sentimientos de culpa, frustración, depresión posparto, ansiedad, arrepentimiento, disconformidad, estrés y preocupación constante (conocida también como "carga mental" y que afecta especialmente a las mujeres cuidadoras): "Si una se ocupa demasiado tiempo en algo, como que igual te sentí culpable, es como: joh no! ¡debería estar haciendo otras cosas! $\mathrm{O}$ a veces me siento culpable por descansar 2 minutos" (Alumna, Campus Juan Gómez Millas Facultad de Filosofía y Humanidades). Estos malestares fueron asociados tanto a las exigencias de la compatibilización de responsabilidades como a aquellos aspectos más subjetivos sobre la maternidad y paternidad siendo estudiantes y jóvenes.

\section{Estrategias de compatibilización}

Existe una noción transversal de que la carga académica en la Universidad de Chile es particularmente alta. Esta exigencia entra en conflicto con la sobrecarga de las responsabilidades parentales, por lo que la posibilidad de destinar todo el tiempo deseado a las responsabilidades universitarias se asume como una imposibilidad desde el principio. Se identifica una estrategia de resignación planificada, ante el diagnóstico de la imposibilidad de rendir todo lo bien que se quisiera por la falta de tiempo, por lo que se decide dedicar el esfuerzo justo y necesario para simplemente pasar los cursos: "En este semestre en todos mis ramos pasó eso, no entregué los últimos trabajos, me pusieron un 1 y tengo promedios 4, 4.5, 4.2. Entonces en realidad calculo mis promedios como justo para poder pasar" (Alumna, Campus Juan 
Campus Gómez Millas - Facultad de Ciencias Sociales). Esto puede traer consigo consecuencias en las expectativas que se generan de cara al futuro con la carrera y las proyecciones profesionales. Otro problema que aparece en este aspecto son las dificultades de coordinación con los tiempos de la universidad, en donde la rigidez de los horarios de clases y entregas de tareas o proyectos aparece como un obstáculo. Frente a esto, dos estrategias utilizadas son hacer los trabajos de forma individual, o a un ritmo distinto respecto del grupo, lo que puede derivar en sobrecarga y dar paso a desacuerdos con sus grupos de trabajo: "No me gusta como que los otros, como predispongan, entonces hago los trabajos solo, de repente los envío tarde por eso mismo, o he faltado a pruebas" (Alumno, Campus Juan Gómez Millas - Facultad de Filosofía y Humanidades). Otra estrategia comúnmente desplegada por estos estudiantes es la superposición de actividades, realizando actividades de diverso tipo en una misma unidad de tiempo. Lo más común es la mezcla de tiempos de cuidado y tiempos de estudio. Esto suele ir acompañado de sensaciones de agotamiento y sentimientos de culpa (sobre todo en mujeres) por no poder reservar un espacio de tiempo específico para la relación con sus hijos/as: "Yo igual estoy con ella, pero es una presencia media ausente haciendo tantas cosas. Lo que más he aprendido a hacer es ser multifuncional en todo momento" (Alumna, Campus Andrés Bello Facultad de Arquitectura y Urbanismo). En ocasiones se despliegan estrategias para aprovechar el tiempo y simultáneamente el disfrute con sus hijos/as, superponiendo tiempos de cuidado y de ocio. Ante jornadas tan exigentes y variadas, una planificación relativamente regular se vuelve necesaria.
La planificación es una de las estrategias más referidas por padres y madres en este caso. La necesidad de organizar rutinas de acción relativamente regulares y gestionar de manera precisa el tiempo aparece como condición para sobrellevar la experiencia cotidiana:

Es como bien del día a día igual, lo que sí intento, para el estudio, aprovechar mucho como las horas que ella está en el jardín, como porque si no implica, cuando ya sale del jardín, estar con ella hasta las 9 o 10, y después estudiar después de las 10, y si es que uno deja todo pal final estay hasta las 3 de la mañana, y al otro día estás cansado (Alumno, Campus Juan Gómez Millas - Facultad de Filosofía y Humanidades). La planificación aparece como un aprendizaje que se consigue con la experiencia de la paternidad, una costumbre aprendida forzosamente a punta de lograr coincidir la agenda académica, personal y familiar. Esto incluso es destacado como una dimensión positiva de la paternidad y como parte del proceso de crecimiento personal. La planificación, sin embargo, choca reiteradamente con la flexibilidad que los cuidados requieren y con la preocupación permanente que la paternidad y la maternidad supone. Las exigencias del cuidado desestructuran la planificación rutinaria, ante lo cual también podemos encontrar la impresión de que no hay forma de organizarse de antemano: "Mi hijo ha tenido accidentes y ha tenido que ir corriendo a ir a verlo o a buscarlo, entonces no sirve mucho planificar la verdad, como que vivo el día a día" (Alumna, Campus Juan Gómez Millas - Facultad de Filosofía y Humanidades). En la mayoría de los casos, el reacomodo de los tiempos deriva en la eliminación total o parcial de actividades recreativas, siendo este un potencial detonante de estrés: "Yo he seguido siendo la misma, he seguido 
militando, he seguido participando de organizaciones sociales, etcétera. Pero si me ha costado el tiempo como de, de distención quizás, como de carretes cachai” (Alumna, Campus Juan Gómez Millas Facultad de Filosofía y Humanidades). Se constata un reacomodo en la cantidad de responsabilidades que se asumen en este tipo de actividades, pasando de tener roles activos (e.g., en organizaciones sociales o políticas) hacia uno secundario. De esta forma, se reafirma que la reducción de objetivos es una estrategia para el proceso material y psicológico de adaptarse a la compatibilización de responsabilidades.

Redes de apoyo

Las redes de apoyo provienen principalmente del grupo familiar, la pareja, instituciones de cuidado $\mathrm{y}$ otras fuentes.

\section{Apoyo familiar}

Es la principal red de apoyo desplegada por estudiantes madres y padres y habitualmente son las mujeres quienes constituyen el mayor soporte de los estudiantes: "Mi mamá trabaja en la casa... y la abuela materna también, y mi hermana también con la universidad tiene horario con los que tiene días libres." (Alumno, Campus Eloísa Diaz - Facultad de Salud). Las abuelas son quienes se hacen cargo del cuidado de sus nietos cuando las madres o padres no pueden, siendo la figura más frecuente como soporte.

El despliegue de las redes de apoyo requiere, además, trasladarse constantemente entre los espacios de estudio, cuidado y delegación del cuidado (i.e., universidad, hogar y jardín/sala cuna), donde el jardín o sala cuna es percibido como una ayuda fundamental y sin la cual la organización horaria y la compatibilización de responsabilidades sería improbable. El soporte familiar es también condición del desarrollo de otras esferas porque supone la liberación de espacios de tiempo que de otra manera serían exclusivamente destinados al cuidado $u$ otras responsabilidades: "Eso me permitió vivir igual lo que se tiene que vivir en esa época. Entonces no fue tan sólo el apoyo de cuidar a XX, sino de permitirme a mí también desenvolverme según la edad que yo tenía" (Alumna, Campus Antumapu - Facultad de Agronomía).

No obstante, el apoyo familiar no está exento de tensiones o complicaciones. La delegación del cuidado genera, en ocasiones, conflictos a nivel intra e interfamiliar. El primero alude a los desacuerdos producto de las decisiones de crianza y división del trabajo de cuidados y los segundos se refieren a conflictos derivados de la necesidad de coordinación entre las familias de la pareja para dividir los tiempos del cuidado y los lugares en donde reside el hijo:

Teníamos que compatibilizar tanto él con su familia y yo el cuidado del niño, ahí tuvimos bastante problemas en la casa. Porque, no sé po', mis papás querían salir con él un fin de semana, pero los papás de él también querían, entonces no lo podía partir en dos" (Alumna, Campus Beauchef - Facultad de Ciencias Físicas y Matemáticas).

\section{Apoyo de la pareja}

La pareja aparece como una importante fuente de apoyo principalmente desde la perspectiva femenina. La información disponible desde la perspectiva masculina es menor, pero indica una distribución 
más equitativa de las actividades de cuidado entre éstos y la madre, independiente de la relación sentimental que tengan: "Fui papá a los 16 años, y al principio claro, los primeros dos años que yo estaba allá, fue muy compartido entre la mamá, sus abuelos, y un poco mi mamá" (Alumno, Campus Juan Gómez Millas - Facultad de Ciencias).

Para el caso de las mujeres, la relación con el padre de su hijo o hija presenta tintes relativamente ambivalentes y con presencia de conflictividad: "Sí, fue súper difícil. Porque yo igual era chica, tenía 17, y fue bastante duro porque él no quería ser papá, entonces yo siempre tenía toda la responsabilidad del niño" (Alumna, Campus Beauchef - Facultad de Ciencias Físicas y Matemáticas). Se encuentran experiencias de escasa o nula presencia paterna, división desigual del trabajo de cuidados o presencia sujeta a la relación sentimental si esta se mantiene: "Hasta hace un año estábamos juntos, entonces me ayudaba harto. Pero, ahora ya no estamos juntos, entonces tampoco le quiero pedir ayuda en cuanto el cuidado del niño" (Alumna, Campus Beauchef - Facultad de Ciencias Físicas y Matemáticas).

Los ejemplos virtuosos de organización entre las parejas se dan de dos formas: bajo la situación en que la pareja sea conviviente y vivan con su hijo, situación en la cual se hay una distribución más equitativa del cuidado; o bien a partir de una organización (diaria o semanal) planificada de la división del tiempo que cada padre/madre destina al cuidado del hijo, situación que también es percibida como compleja: "A veces es super complicado porque él trabaja de noche, entonces... Por ejemplo, ahora tuvo libre y se pudo quedar con mi hija, o sino no habría podido venir" (Alumna, Campus Andrés Bello - Facultad de Economía y Negocios). No se encuentran casos de madres que puedan llevar a cabo por sí solas todas estas labores sin un apoyo de terceros.

\section{Instituciones de cuidado}

Organismos como el jardín infantil, sala cuna, y servicios remunerados también son considerados como un importante apoyo. Los jardines infantiles aparecen como el soporte más importante después de la familia. Habitualmente constituyen un espacio que libera un tiempo destinado a las actividades académicas (i.e., asistencia a clases principalmente). No obstante, a veces se presentan conflictos con estas instituciones, lo que se relacionan principalmente con desacuerdos en el plano de los ideales educativos, jornadas muy extensas (a veces por necesidad del padre o madre, lo que deriva en sentimientos de culpa) o temas de coordinación del tiempo (e.g., horarios de entrada y salida). Sin embargo, este apoyo suele verse como beneficioso y necesario.

Los servicios remunerados particulares, como trabajadoras de casa particular o cuidadoras, son menos frecuentes, por razones contingentes o para momentos específicos:

En situaciones que he necesitado dejar a mi hijo y que ninguno de los dos ha podido, o cuando me han llamado para alguna urgencia del jardín, he tenido que decirle a la señora que cuida a mis hermanos, que lo vaya a buscar, y nosotros tenemos que pagarle a ella por horas (Alumna, Campus Juan Gómez Millas - Facultad de Ciencias Sociales). 


\section{Otras redes de apoyo}

Resulta interesante notar que las amistades no suelen presentarse como alternativas de cuidado. A pesar de ser algo valorado en el plano emocional, solicitar su apoyo en los cuidados puede aparecer como no deseado. En este aspecto, se genera un desajuste en torno a la noción de madurez de los pares, no confiando en dar a sus amigos la responsabilidad de su hijo. La noción de madurez es relevante pues describe el desarrollo y crecimiento personal que supone la paternidad-maternidad, como condicionante para confiar el cuidado del hijo:

No sé, igual tenemos 24 , son más inmaduros como en ese aspecto. No le dejaría mi hija a un amigo, la verdad (...) no tengo ningún amigo que sea papá, entonces no, en ese sentido estoy solo. Pero me ayudan con las pruebas, como a estudiar, cosas así" (Alumno, Campus Eloísa Diaz - Facultad de Salud).

También aparece la figura de las vecinas como personas que entregan un apoyo esporádico. A pesar de que no son una fuente regular de soporte, su presencia es importante para resolver problemas de coordinación o para liberar espacios de tiempo que de otra manera no podrían existir, especialmente tiempo libre o de dispersión.

Finalmente, la institución universitaria también aparece como una red de apoyo en distintos niveles. La promulgación de la política de corresponsabilidad social en los cuidados de la Universidad de Chile permite flexibilidades académicas y de asistencia, ayudas económicas y la implementación de salas cunas o jardines infantiles en las distintas unidades administrativas. Sin embargo, se constató que su implementación aún está fuertemente mediada por la disposición de los actores universitarios que son parte del proceso. Respecto a las y los académicos se señalan experiencias donde empatizan con la situación de maternidad y paternidad de los estudiantes, no obstante, gran parte de las personas entrevistadas cuentan con alguna mala experiencia, las cuales tienden a repetirse con académicos hombres. Desde dificultades en las flexibilidades de asistencia, penalización en las calificaciones, hasta comentarios discriminatorios:

En muchos casos empatizan, pero tampoco es como que tengan flexibilidad en la entrega de trabajos, $o$ si una tiene que faltar a una prueba, igual te la hacen más difícil (Alumna, Campus Andrés Bello - Facultad de Arquitectura y Urbanismo).

Yo creo que debería haber alguien que fiscalizara todas las evaluaciones orales, porque a mí me ha pasado que me han humillado delante de 50 personas en una evaluación oral porque no voy nunca. Entonces me han dicho si no encuentro un poco perverso usar como excusa mi hijo para no ir a clases. (Alumna, Campus Andrés Bello - Facultad de Derecho).

Los funcionarios no académicos de la universidad se perciben como los agentes más facilitadores, por medio de la entrega de información, ayuda en procesos burocráticos y mediación ante problemas de diversa índole. Por su parte, los compañeros de facultad y organizaciones estudiantiles se señalaron como relevantes para difundir los beneficios estudiantiles de la universidad y apoyar en el ámbito académico, sin embargo, también se señaló en varias oportunidades la existencia de poca empatía en situaciones cotidianas de la vida universitaria, tales como el desarrollo de las clases expositivas en horarios que les dificultan, trabajos en grupo, entre otras situaciones. 


\section{CONCLUSIONES}

De los resultados de este estudio se puede concluir que las madres y los padres que cursan estudios universitarios poseen extensas jornadas diarias de trabajo, en donde las responsabilidades relativas a su condición de maternidad o paternidad se entrecruzan con el resto de sus responsabilidades académicas, domésticas y/o laborales. Estas jornadas también se caracterizan por tener una alta variabilidad, debido a que están sujetas a cambios constantes relacionados a las actividades del cuidado, por lo que una planificación con altos niveles de flexibilidad resulta fundamental para lograr compatibilizar sus actividades.

Hay también una constante superposición de actividades. Las distintas responsabilidades son realizadas al mismo tiempo e incluso en los mismos espacios físicos, lo que dificulta la posibilidad de que estas personas puedan desconectarse de las mismas. Además, en la mayor parte de los casos se señalaron dificultades para poder tener espacios de esparcimiento e incluso dificultades para el cumplimiento de sus necesidades básicas (e.g., alimentación, descanso, cuidado personal, etc.). En efecto, una de las estrategias más utilizadas para la compatibilización de las responsabilidades de madres y padres, es la supresión de los tiempos de esparcimiento o de necesidades básicas para priorizar el cumplimiento de sus variadas responsabilidades. Las cinco estrategias para el ejercicio de los cuidados detectadas por Arriagada (2010) se repiten constantemente como fórmulas para compatibilizar responsabilidades, añadiendo la diferenciación de estrategias según nivel de responsabilidad en los cuidados y los sesgos por roles de género, cuestiones señaladas anteriormente.

Lo anterior genera que madres y padres manifiesten frecuentemente sensación de estrés, desequilibrio emocional y sentimiento de culpa sobre el (in)cumplimiento de sus tareas del día a día. Esto también dificulta su buen rendimiento académico, lo cual muestra a la universidad como un espacio poco pensado para la compatibilización con los cuidados y la crianza ya que implica alta carga académica, poca empatía por parte de otros estamentos y plazos para la entrega de evaluaciones con los que cuesta cumplir. De esta manera, se terminan implementando estrategias que merman el desarrollo individual de los y las estudiantes, tales como la reducción de objetivos de vida o la resignación planificada respecto a su rendimiento académico, que trae consigo un impacto negativo en su salud mental.

Se observaron diferencias en la experiencia de madres y padres, derivada de cuestiones relativas a las expectativas que se tienen de ellos según roles de género socialmente asignados. Por una parte, hay mayor incentivo en terminar la carrera para padres, con el fin de obtener el título y con ello la entrada al mundo laboral, por otra parte, las exigencias en torno a las labores de cuidado y crianza son mayores para las mujeres, cuestión que se hace extensiva a miembros femeninos de la familia. Esto genera una diferencia importante en torno al tipo de estrategias de compatibilización de las distintas actividades que tienen los y las estudiantes. Además, se observaron fuertes cuestionamientos 
a lógicas de diferenciación sexual, ya sea por madres que persiguen mayores niveles de empoderamiento sobre su vida personal y profesional, o por padres que buscan asumir de manera formal sus labores de crianza y en igualdad de responsabilidades, cuestión que se podía observar también, aunque de manera incipiente, en los resultados de la investigación de Castañeda (2015). Estos cuestionamientos, sin embargo, no suelen ser suficientes para modificar las estructuras de diferenciación sexual en torno a los cuidados.

Respecto a alcances metodológicos, mencionar que en primera instancia se realizaron grupos focales pues se buscaba que los participantes pudiesen dialogar desde sus experiencias en torno a la maternidad/paternidad universitaria. Posterior a su realización, se optó por utilizar entrevistas en profundidad semi estructuradas, ya que la coordinación de los distintos grupos focales resultó sumamente dificultosa, por los tiempos acotados y dificultades varias que tienen estudiantes madres y padres, cuestión que se encuentra en la misma línea de los hallazgos del estudio.

Acerca de posibles líneas de investigación a futuro, se considera interesante el poder profundizar en la gran diversidad de las experiencias en torno a la crianza y vida universitaria de estudiantes madres y padres. La investigación develó diferencias especialmente vinculadas a cuestiones de género (experiencias diferenciadas según maternidad o paternidad) o por cuestiones relativas a los distintos niveles de implementación en los campus de la política de corresponsabilidad social en los cuidados de la Universidad de Chile. Sin embargo, también fueron reveladas otras diferencias que no fueron el foco inicial de la investigación, tales como las implicancias que tienen las diferencias socioeconómicas o su situación de pareja.

Finalmente, los resultados aportan en la línea de la necesidad de adaptar las instituciones y el mercado laboral "a las demandas de la crianza, creando estructuras y criterios adecuados para las madres y para que todas y todos tengamos mucho más tiempo para cuidar" (Merino, 2018, p.74), factores que resultan imprescindibles para garantizar la igualdad de género y el bienestar general humano. A la luz de los acontecimientos mundiales de la actualidad (e.g., crisis del sistema político económico neoliberal, revueltas sociales por redistribución de la riqueza, el confinamiento a la vida doméstica por la situación de pandemia, y el auge y expansión del movimiento feminista) los planteamientos en torno a la corresponsabilidad social de los cuidados toman mayor relevancia para repensar nuestra sociedad.

\section{REFERENCIAS}

Araneda Guirriman, C., Gairín Sallán, J., Pedraja-Rejas, L., \& Rodríguez-Ponce, E. (2018). Percepciones sobre el perfil del estudiante universitario en el contexto de la educación superior de masas: Aproximaciones desde Chile.
Interciencia. Revista de Ciencia y Tecnología de las Américas, 43(12), 864-870. Recuperado de http://www.interciencia.net/

Arriagada, I. (2010). La crisis de cuidado en Chile. Revista de Ciencias Sociales, 23(27), 58-67. 
Álvarez Moral, D., Guerra Maldonado, C., Tapia Sepúlveda, K., \& Varas González, G. (2020). Compatibilizando estudios y familia: Ser estudiante y a la vez madre o padre. Revista de Sociología, 35(2), 61-77. doi: 10.5354/0719-529X.2020.58647

Canales, A., \& De los Ríos, D. (2009). Retención de estudiantes vulnerables en la educación universitaria. Revista Calidad en la Educación, (30), 50-83. doi: 10.31619/caledu.n30.173

Carrasco, C. (2013) El cuidado como eje vertebrador de una nueva economía. Cuadernos de relaciones laborales, 31(1), 39-56. doi: 10.5209/rev_CRLA.2013.v31.n1.41627

Castañeda, M. (2015) Ser estudiantes, madres y padres: Una dualidad cotidiana [Tesis de licenciatura, Universidad de Chile]. Recuperado de http://repositorio.uchile.cl/

Clark, W. (2006). Academic charisma and the origins of the research university. Chicago, MI: University of Chicago Press.

Dides, C., \& Fernández, C. (Eds.). (2016). Salud sexual, salud reproductiva y derechos humanos en Chile (Primer informe estado de la situación 2016). Santiago, Chile: Corporación MILES.

Dirección de Bienestar y Desarrollo Estudiantil (DIRBDE). (2019). Estudiantes madres y padres en la Universidad de Chile. Recuperado de http://gestiondbe.uchile.cl/menus/docs/madr es_padres.pdf

Donoso, S., \& Schiefelbein, E. (2007). Análisis de los modelos explicativos de retención de estudiantes en la universidad: Una visión desde la desigualdad social. Estudios Pedagógicos, 33(1), 7-27. doi: 10.4067/S0718-07052007000100001

Federici, S. (2013). Revolución en punto cero. Trabajo doméstico, reproducción y luchas feministas. Madrid, España: Traficantes de Sueños.

Gaínza, A. (2006). La entrevista en profundidad individual. En M. Canales (Comp.), Metodologías de investigación social. Introducción a los oficios (pp.219-263). Santiago, Chile: LOM Ediciones.

Hernández, R., Fernández, C., \& Baptista, P. (2010). Metodología de la investigación (5 ${ }^{\mathrm{a}}$ ed.). México, D.F.: Mc Graw Hill.

Huerta, E. (2018). Consejo Universitario aprueba reglamento de corresponsabilidad social en el cuidado de hijos de estudiantes de la Universidad de Chile. Universidad de Chile. Recuperado de http://uchile.cl/u141300

Instituto Nacional de Estadísticas (INE). (2017). Enfoque estadístico. Estadísticas vitales sobre maternidad y paternidad adolescente (Documento de trabajo, indicadores de género y metodologías INE). Santiago, Chile: Instituto Nacional de Estadísticas.

Martinic, S. (2006). El estudio de las representaciones y el análisis estructural de discurso. En M. Canales (Comp.), Metodologías de investigación social. Introducción a los oficios (pp.299-348). Santiago, Chile: LOM Ediciones.

Merino, P. (2018). La maternidad como cuidado: Guía hacia un futuro sostenible. Revista Viento Sur, (156), 69-77. Recuperado de http://vientosur.info/category/revista/156/

Moscovici, S. (1961). La psychanalyse: Son image et son public. París, Francia: Presses Universitaires de France.

Pateman, C. (1995). El contrato sexual. Barcelona, España: Editorial Anthropos.

PNUD. (2012). Desarrollo humano en Chile 2012. Bienestar subjetivo: El desafío de repensar el desarrollo. Santiago, Chile: PNUD 
Álvarez Moral, D., Guerra Maldonado, C., Tapia Sepúlveda, K., \& Varas González, G. (2020). Compatibilizando estudios y familia: Ser estudiante y a la vez madre o padre. Revista de Sociología, 35(2), 61-77. doi: 10.5354/0719-529X.2020.58647

Rivas Ureta, G. (2010). El impacto de la paternidad y maternidad en jóvenes de clase media. Cambios y resistencias en los roles/identidades de género [Tesis de licenciatura, Universidad de Chile]. Recuperado de http://repositorio.uchile.cl/

Servicio de Información de Educación Superior (SIES). (2019). Informe matrícula 2019 en educación superior en Chile. Recuperado de http://www.mifuturo.cl/

Strauss, A., \& Corbin, J. (2002). Bases de la investigación cualitativa. Técnicas y procedimientos para desarrollar la teoría fundamentada. Medellín, Colombia: Editorial Universidad de Antioquia.

Universidad de Chile. (2018) Política de corresponsabilidad social en la conciliación de las responsabilidades familiares y las actividades universitarias. Santiago, Chile. Recuperado de

http://repositorio.uchile.cl/handle/2250/149313

Valdés, X. (2004). Familias en Chile: Rasgos históricos y significados actuales de los cambios. En I. Arriagada \& V. Aranda (Comps.), Cambios de las familias en el marco de las transformaciones globales: Necesidad de políticas públicas eficaces (pp. 335-353). Santiago, Chile: CEPAL.

Wilkis, A. (2004) Apuntes sobre la noción de estrategia en Pierre Bourdieu. Revista Argentina de Sociología, 2(3), 118-130. Recuperado de http://www.redalyc.org/pdf/269/26920307.pdf

Manuscrito recibido: 10-07-2020

Manuscrito aceptado: 21-09-2020 